\title{
COMPLICAÇÕES FÍSICAS E PSICOLÓGICAS VIVENCIADAS PELAS MULHERES EM CONSEQUÊNCIA DA INDUÇÃO DO ABORTO CLANDESTINO: UMA REVISÃO INTEGRATIVA
}

\author{
PHYSICAL AND PSYCHOLOGICAL COMPLICATIONS EXPERIENCED \\ BY WOMEN AS A RESULT OF THE INDUCTION OF CLANDESTINE \\ ABORTION: AN INTEGRATIVE REVIEW
}

\author{
Cíntia Silva Oliveira ${ }^{1}$ \\ Maria Stefania Nóbrega Batista ${ }^{2}$
}

\begin{abstract}
RESUMO: Introdução: A interrupção da gravidez só é considerada aborto quando acontece antes das vinte e duas semanas de gestação e se o peso do feto for inferior a 500mg. Estima-se que, anualmente, cerca de 46 milhões de abortos são realizados no mundo; desses, cerca de 19 milhões são ilegais. Há muitas complicações possíveis e frequentes com a indução do aborto inseguro. Anualmente, cerca de 68 mil mulheres morrem e outras 5,3 milhões desenvolvem sequelas, físicas ou psicológicas, em consequência dessa prática. Objetivo: Identificar, analisar e correlacionar as complicações físicas e psicológicas vivenciadas pelas mulheres em consequência da indução do aborto clandestino. Metodologia: Foi realizada uma revisão integrativa da literatura baseada nas bibliotecas virtuais SCIELO, BVS, PUBMED, MEDLINE e LILACS norecorte temporal entre 2015 e 2020, incluindo os idiomas inglês, português e espanhol. No estudo, foram identificados 5503 artigose, após aplicados os critérios de inclusão e exclusão, 20 artigos foram selecionados. Resultados: A indução do aborto clandestino causa diversas complicações físicas e psicológicas. Dentre as físicas, destacam-se as hemorragias, infecções, perfuração uterina, sepse e óbito. Em relação às psicológicas, temos como principais o constragimento, tristeza, sentimento de culpa e estresse emocional. Conclusão: As complicações físicas e psicológicas estão muito presentes na realidade das mulheres que induziram 0 aborto clandestino. Contudo, tais complicações podem ser diminuídas ou até mesmo evitadas a partir de um atendimento por equipe médica qualificada.
\end{abstract}

\footnotetext{
1 Graduanda em Medicina. Centro Universitário de Patos - UNIFIP, Patos, Paraíba, Brasil.cintiaoliveira@med.fiponline.edu.br.

${ }_{2}^{2}$ Especialista em Ginecologia, Obstetrícia e Mastologia. Centro Universitário de Patos - UNIFIP, Patos, Paraíba, Brasil.stefania_batista@hotmail.com.
} 
Palavras chave: Mulheres. Complicações. Indução do aborto.

\begin{abstract}
Introduction: Termination of pregnancy is only considered a miscarriage when it takes place before the twenty-two weeks of gestation and if the fetal weight is less than 500mg.lt is estimated that, annually, about 46 million abortions are performed worldwide; of these, about 19 million are illegal.There are many possible and frequent complications with the induction of unsafe abortion. Annually, about 68 thousand women die and another 5.3 million develop sequelae, physical or psychological, because of this practice. Objective: Identify, analyze and correlate the physical and psychological complications experienced by women because of the induction of clandestine abortion. Methodology: An integrative literature review was performed based on the SCIELO, BVS and PUBMED, MEDLINE and LILACS virtual libraries in the period between 2015 and 2020, including English, Portuguese and Spanish.In the study, 5503 articles were identified and, after applying the inclusion and exclusion criteria, 20 articles were selected. Results: Induction of clandestine abortion causes several physical and psychological complications. Among the physical, hemorrhages, infections, uterine perforation, sepsis and death stand out.Regarding the psychological ones, the main ones are embarrassment, sadness, guilt and emotional stress.Conclusion: Physical and psychological complications are present in the reality of women who induced clandestine abortion. However, such complications can be reduced or even avoided, through care provided by a qualified medical team.
\end{abstract}

Keywords: Women. Complications. Inductionofabortion. 


\section{INTRODUÇÃO}

A interrupção da gravidez só é considerada aborto se for realizada antes das vinte e duas semanas de gestação e se o peso do feto for inferior a $500 \mathrm{mg}$, ou seja, se o concepto for incapaz de sobreviver fora do corpo da mãe. Existem dois tipos principais de aborto, o espontâneo e o induzido; podendo, este último, ser legal ou clandestino, a depender do motivo e do país em que será realizado (SELL et al., 2015).

O aborto induzido é um assunto bastante polêmico, pois envolve aspectos morais e religiososda população. No Brasil, por exemplo, o aborto só não é considerado crime em casos de risco de vida para a mulher, gestação resultantede estupro ou se o feto for anencefálico. Devido a isso, muitas mulheres recorrem à prática do aborto em condições inseguras e insalubres (SELL et al., 2015).Estima-se que, anualmente, 46 milhões de abortos são realizados no mundo, destes, cerca de 19 milhões são ilegais. As maiores taxas são identificadas em nações subdesenvolvidas e em desenvolvimento, como na África, países da América Latina, Caribe e o Sul e Sudeste Asiáticos (ZAFAR et al., 2018).

A indução do aborto inseguro é um grande fator de desigualdade, pois as mulheres que se submetem a tal procedimento são pobres, jovens,de raça negra, com baixa escolaridade, condições precárias de moradia e, ao mesmo tempo, têm mais filhos, menos parceiros fixos e são mais frequentemente as principais responsáveis pela família. Dessa forma, as mulheres que não possuem condições financeiras que garantam acompanhamento adequado em clínicas e consultórios privados recorrem ao uso de medicamentos obtidos de forma clandestina e ilegal, como o misoprostol. Em geral, após início do sangramento, elas procuram assistência hospitalar pública para finalizar o processo e tratar possíveis complicações (GOES et al., 2020).

De acordo com Zafaret al.(2018), a prática antiética e ilegal por profissionais de saúde ou curandeiros tradicionais não treinados e não qualificados, 
complementada por padrões de higiene precários e falta de experiência, causa complicações graves. Há muitas complicações possíveis e frequentes com a indução do aborto inseguro. Estima-se que, anualmente, 68 mil mulheres morrem e outras 5,3 milhões apresentam sequelas em consequência dessa prática. Essas complicações podem ser transitórias ou permanentes a depender da gravidade. São exemplos delas: hemorragias, choque, infecções, sepse, perfurações, falência de órgãos, doença inflamatória pélvica crônica e infertilidade (ZAFAR et al., 2018).

Após a realização do aborto, muitas mulheres podem desenvolver problemas psicológicos, sendo tomadas pelos sentimentos de negação, culpa, raiva, medo e ressentimento. Em casos graves, uma condição pode haver mais severa, a síndrome pós-aborto, sendo os principais sintomas insônia ou perturbações do sono, pesadelos constantes, síndrome do pânico e, até mesmo, ideação suicida. Em alguns casos, essa culpa pode, de certa forma, consumir a vida da mulher enfaticamente, fazendo com que ela procure meios de "consertar" o aborto realizado, mediante uma nova gestação por exemplo(VALADÃO; REIS; ROCHA, 2019).

Foi observado, em um estudo realizado em Portugal,que antesda descriminalização do aborto ser aprovada, o número estimado de abortos realizados na população portuguesaera de 20 mil por ano. Após a nova lei entrar em vigor, a taxa nunca ultrapassou os números anteriores. Levando em consideração os dados dos países europeus, o número de abortos por mil nascidos vivos, em Portugal, manteve-se abaixo da média após a descriminalização (VICENTE, 2020).

Essa pesquisa tem como finalidadesalientar que, devido ao aborto ser ilegal em grande parte dos países, os ambientes em que são realizados não possuem condições adequadas e as pessoas que os realizam não são qualificadas para exercer tal função, ocasionandonúmero elevados de complicações físicas e psicológicas, muitas vezes graves e irreversíveis.

É importante destacar que a descriminalização não eleva o número de abortos realizados, mas ajuda a diminuir o número de morbimortalidade das mulheres, que são assistidas de forma correta e em ambientes adequados à realização dos procedimentos. 
Destarte, esta revisão visou identificar complicações físicas e psicológicas vivenciadas pelas mulheres em consequência da indução do aborto clandestino. Ademais, realizou-se uma comparação entre as taxas de mortalidade feminina de países que possuem o aborto legalizado com os que não possuem, sendo feita uma análise do perfil socioeconômico das mulheres que realizam o aborto induzido clandestino, e as principais causas que as impulsionaram.

\section{OBJETIVO}

Identificar, analisar e correlacionar as complicações físicas e psicológicas vivenciadas pelas mulheres em consequência da indução do aborto clandestino.

\section{METODOLOGIA}

A Revisão Integrativa da Literaturaidentifica, analisa e sintetiza os resultados de pesquisas de um determinado assunto. Para a sua formulação são realizadas seis fases, sendo elas: (1) elaboração da pergunta norteadora; (2) busca ou amostragem na literatura; (3) coleta de dados; (4) análise crítica dos estudos incluídos; (5) discussão dos resultados; e (6) apresentação da revisão integrativa (SOUZA; SILVA; CARVALHO, 2010).

$\mathrm{Na}$ primeira etapa, a pergunta norteadora do estudo foi: quais as complicações físicas e psicológicas vivenciadas pelas mulheres em consequência da indução do aborto clandestino?

A segunda etapa consistiu na estratégia de busca, na qual foram utilizados os descritores em Ciências da Saúde (DeCS), juntamente com o operador booleano "AND", que originou o descritor: "Women" AND "Complications" AND "Inducedabortion". 
$\mathrm{Na}$ terceira etapa, o processo de busca e seleção dos artigosocorreu nas bibliotecas virtuais: US National Library of Medicine National Institutes of Health (PUBMED), The Scientific Electronic Library Online (SCIELO), Literatura LatinoAmericana e do Caribe em Ciências da Saúde (LILACS) e Medical Literature Analysis and Retrieval System Online (MEDLINE). Esses dois últimos, acessados pela Biblioteca Virtual em Saúde (BVS). Ademais, foram selecionados artigos nos idiomas inglês, português e espanhol, em recorte temporal entre 2015 e 2020. Os artigos que não se enquadraram nos objetivos e os duplicados foram excluídos.

Mediante a identificação dos artigos, a quarta etapa consistiu na leitura crítica dos 20 artigos, análise e interpretação dos seus principais achados na tentativa de responder a questão norteadora. No estudo, as variáveis selecionadas foram: título do artigo, autores, base de dados, periódicose ano da puplicação. A discussão dos principais resultados encontrados foi efetuada na quinta etapa. E a sexta, constituiuse na apresentação da revisão integrativa.

\section{RESULTADOS}

A indução do aborto clandestino causa diversas complicações físicas e psicológicas. Dentre as físicas, destacam-se as hemorragias, infecções, perfuração uterina, sepse e óbito. Em relação às psicológicas, temos como principais o constragimento, tristeza, sentimento de culpa e estresse emocional.

\section{DISCUSSÃo}

Conforme os resultados analisados, foi possível verificar as principais complicações físicas (DOMINGUES et al., 2020., AKINLUSI et al., 2018., MELESE et al., 2017., VOLKOV et al., 2018., NAHAR et al., 2017., ISHOSO et al., 2019., ADANIKIN et al., 2019., ZAFAR et al., 2018., KATUASHI; TSHEFU; COPPIETERES, 
2018., GAROFALO et al., 2018., PRADA et al., 2015) e psicológicas (ARAUJO et al., 2018., DOMINGUES et al., 2020., MADEIRO; RUFINO, 2017) vivenciadas pelas mulheres em consequência da indução do aborto clandestino, levando em consideração o perfil socioeconômico das pacientes e quais foram osmotivos mais importantes que as impulsionaram.

A maioria das mulheres reconheceu a necessidade de cuidados após a prática do aborto clandestino, mas demoraram, em média, 10 horas desde os primeiros sintomas até a procura de atendimento. Dificuldade de acesso a meios de transporte rápidos e diretos, indisponibilidade de locais para deixarem os outros filhos eobtenção de licença do trabalho (ARAÚJO et al., 2018) foram os principais limitantes.

Ademais, muitos atrasos ocorreram por medo, vergonha e/ou constrangimento, pois muitos relatos de atendimento desumanizado, desrespeitoso, abusivo e de comportamento crítico por profissionais de saúde são documentados em vários lugares (ARAÚJO et al., 2018).

Os maiores níveis da prática do aborto inseguro, no Brasil e em outros países subdesenvolvidos ou em desenvolvimento, estão entre as mulheres de raça negra, de baixa renda e escolaridade, crianças, adolescentes e jovens em situação de rua, profissionais do sexo e usuárias de álcool e drogas. Tem-se observado que o aborto serviriacomo forma de regulação da procriação na ausência ou falha de métodos contraceptivos(DOMINGUES et al., 2020).

A morbimortalidade materna causada pelo aborto inseguro é maior que a do aborto seguro, além de ser mais presente em países de baixa e média rendas. As estimativas mais recentes indicam que o primeiro é responsável por 9,9\% dos óbitos maternos na América Latina e Caribe (DOMINGUES et al., 2020).

Um dos maiores motivos para esse aumento da morbimortalidade é o atraso na assistência obstétrica adequada, pois a mulher não encontra apoio social, possui receio de assumir uma prática ilegal e tem dificuldade de acesso aos serviços de saúde (DOMINGUES et al., 2020).

Desfechos negativos na saúde mental podem ser verificados em mulheres que não obtiveram êxito na interrupção da gravidez, como:comportamento suicida, depressão e ansiedade. Geralmente as mulheres que desenvolvem sintomas de 
estresse pós-traumático pós-aborto induzido são jovens, de menor escolaridade, com níveis mais elevados de ansiedade e depressão e maior necessidade de aconselhamento (DOMINGUES et al., 2020).

Há diversas formas de induzir o aborto. Aintrodução de objetos pelo trato genital é muito habitual, podendo causar danos no intestino e no sistema reprodutivo, provocando dores abdominais, febre, sangramentos, anemia, sepse e morte (AKINLUSI et al., 2018).

Devido ao estigma do aborto, muitas mulheres retardam a procura de assistência médica, fato que pode implicar em infertilidade, dor pélvica crônica, gestações ectópicas ou até mesmo a morte materna (AKINLUSI et al., 2018).

Existem semelhanças entre 0 aborto espontâneo e o induzido, porém esse último possui características específicas, comocorrimento vaginal (sinal mais comum de infecção), sensibilidade pélvica e choque séptico, perfuração uterina e perfuração intestinal com peritonite generalizada. Muitas dessas complicações poderiam ter suas intensidades diminuídas ou, até mesmo, serem evitadas se a apresentação das pacientes ao sistema de saúde não fosse tardia (MELESE et al., 2017).

A comparação de estudos realizados antes e após a legalização do aborto, na África do Sul, evidenciou que houve uma redução moderada nas complicações de alta gravidade, ou seja, abortos assistidos causam menos danos às mulheres e, consequentemente, custam menos para o governo do que o tratamento de complicações da interrupção da gravidez clandestina (MELESE et al., 2017).

É frequente o retardo na procura por assistência médica nos países em que a realização do aborto é ilegal, principalmente devido a não prática do sigilo na relação médico-paciente e à falta de ética, de modo geral, por parte dos profissionais de saúde (LABANDERA; GORGOROSO; BRIOZZO, 2016).

Nações em que a interrupção da gravidez não é considerada um crime, possuem, em sua maioria, profissionais e equipes de saúde quereconhecem o compromisso profissional e a obrigação de garantir o sigilo e a confidencialidade médica, proporcionando uma diminuição considerável das complicações do aborto inseguro e, consequentemente, da mortalidade materna. (LABANDERA; GORGOROSO; BRIOZZO, 2016). 
A mortalidade materna em consequência do aborto clandestino possui duas principais causas, a mais prevalente é a sepse, juntamente com a falência múltipla dos órgãos, seguida por hemorragias. Levando em consideração esses dados, os dois procedimentos mais utilizados nestes casos são a curetagem repetida da cavidade uterina e cirurgia posterior (VOLKOV et al., 2018).

É habitual o pensamento de que políticas antiaborto ajudariam a reduzir o número dessa prática em todo o mundo, entretanto, as evidências mostram aumento significativo desses valores. Outrossim, quando o aborto é legal, podemos reduzir a morbimortalidade devido aos cuidados de saúde adequados,a educação sobre sexualidade, o acesso a anticoncepcionais, atenção pós-aborto e intervenções adequadas (HUG et al., 2017).

Um estudo realizado na Dhaka Medical College Hospitalevidenciou que de 50 casos de abortos sépticos, 44 foram induzidos e a maioria deles aconteceu em mulheres do lar, que moravam com seus cônjuges e possuíam baixa escolaridade. A maioria das mulheres não queria mais filhos e optou por interromper a gravidez para não sobrecarregar o lar ou por possuir um relacionamento conjugal perturbado (NAHAR et al., 2017).

Dentre os 44 abortos sépticos induzidos, as principais causas de óbito observadas foram peritonite generalizada com septicemia, septicemia com insuficiência renal eaborto séptico com coagulação intravascular disseminada. Ademais, foi observado que o uso de anticoncepcionais e a educação sexual são fundamentais para que a incidência de abortos sépticos e suas complicações sejam reduzidas (NAHAR et al., 2017).

Em pesquisa realizada no Zimbábue, África,foi possível identificar que mulheres jovens, moradoras da zona rural, solteiras, com baixo nível de escolaridade e que já possuíam filhos eram as com maiores taxas de complicações pós-aborto clandestino (MADZIYIRE et al., 2018).

A política nacional do Ministério da Saúde e Assistência Infantil do Zimbábuepreconiza que a atenção pós-aborto (APA) deve ser gratuita em instalações públicas, porém é necessário que seja pago o transporte, taxas adicionais e outras despesas. Em consequência, muitas mulheres demoram a 
procurar o serviço de saúde, favorecendo a piora de suas complicações (MADZIYIRE et al., 2018).

Destarte, melhorar a acessibilidade de mulheres mais pobres aos serviços de APAé essencial para diminuir a mortalidade materna, uma vez que essa população possui maior probabilidade de realizar abortos inseguros. O misoprostol e a aspiração manual intrauterina (AMIU) deveriam ser fornecidos pelos centros de saúde primários; assim, haveria maior acessibilidade e menor congestionamento das instalações de nível superior, com provável redução da mortalidade materna (MADZIYIRE et al., 2018).

Complicações psicológicas pós-aborto são bastante comuns. Um dos motivos é a internação em unidades conjuntas com puérperas e seus recém-nascidos. De acordo com um estudo brasileiro, mal-estar emocional, tristeza, constrangimento, frustração e piora do sentimento de culpa foram declarações comuns (MADEIRO; RUFINO, 2017).

Diante do fato de que o aborto não é legalizado no Brasil, a ausência de espaços separados para atender às especificidades de mulheres pós-aborto pode significar mais uma forma de discriminação, punição e violência institucional (MADEIRO; RUFINO, 2017).

Estudo realizado na Costa do Marfim mostrou que as mulheres que faziam uso de métodos contraceptivos tinham maior risco de abortar, poisrefletiria o desejo de não quererem engravidar. Além do uso de anticoncepcionais, outros fatores influenciam no aumento do risco de aborto, comoidade mais jovem, níveis mais altos de educação e sexo transacional (sexo em troca de presentes ou dinheiro) (RATOVOSON et al., 2020).

Análises feitas em Kinshasa, capital da República Democrática do Congo,evidenciaram uma correlação significativa entre o uso excessivo de bebidas alcoólicas e a incidência de aborto induzido. O consumo exagerado pode afetar diretamente a decisão de abortar ou levar a relações sexuais desprotegidas, podendoresultar em gravidez indesejada. Além disso, houve associação significativa com mulheres solteiras (separadas, divorciadas ou viúvas), debaixo nível escolar e com o contexto social de Kinshasa, em que a gravidez fora do casamento não é tolerada (ISHOSO et al., 2019). 
Complicações foram observadas principalmente em distritos excêntricos e semirrurais da capital, evidenciando que o baixo nível socioeconômico limita a compra de medicamentos para cuidados pós-aborto, como também indica abaixa qualidade dos procedimentos realizados nestes bairros. A hemorragia foi o tipo de complicação prevalente, seguida por infecção e trauma genital (ISHOSO et al., 2019).

Apesar de a hemorragia ser a complicação mais frequente, a infecção (sozinha ou em combinação com a hemorragia) causou mais mortes. Portanto, é essencial que os profissionais de saúde da linha de frente considerem a sepse com antecedência em mulheres que apresentam queixas relacionadas ao aborto e, consequentemente, tomem as medidas adequadas (ADANIKIN et al., 2019).

Mulheres com níveis educacionais mais elevados, correlacionado com o status socioeconômico, tiveram menores taxas de mortalidade pós-aborto, pois conseguiram identificar os "sinais de perigo" de forma precoce e foram capazes de pagar pelas intervenções necessárias (ADANIKIN et al., 2019).

Em países onde o aborto não é legalizado, a maioria das mulheres com gravidez indesejada ingerem abortivos não recomendados e procuram profissionais de saúde ou curandeiros tradicionais não treinados e não qualificados. Correlacionado a isso, há os padrões de higiene precários e falta de experiência. Por esse motivo, complicações são muito comuns, como hemorragias, choque, infecções, sepse, perfurações, falência de órgãos, doença inflamatória pélvica crônica, infertilidade ou mesmo morte (ZAFAR et al., 2018).

A perfuração intestinal foi a complicação mais comum, levando em consideração apenas o aborto induzido. A mais grave foi a perfuração uterina com ou sem comprometimento intestinal. O útero localiza-se próximo ao sigmoide e ao reto; o aumento do volume uterino devido à gestação torna esses dois órgãos mais suscetíveis a lesõesna prática do aborto (ZAFAR et al., 2018).

Em um estudo comparativo, foi observado que as pacientes com complicações relacionadas ao aborto induzido tiveram permanências hospitalares significativamente mais longas do que as com outras complicações obstétricas e ginecológicas. Em relação às características dos óbitos, foi identificado um risco aumentado para pacientes com pelviperitonite pós-aborto em comparação com 
outras emergências ginecológicas e obstétricas. (KATUASHI; TSHEFU; COPPIETERS, 2018).

Uma pesquisa realizada emSant'anna Hospital ofTurinUniversity, Itália, constatou que as complicações mais prevalentes, em decorrência do aborto induzido, são hemorragias, infecções, riscos de retenção placentária pósprocedimento, ruptura uterina, histerectomia e necessidade de realização de cesariana (GAROFALO et al., 2018).

Em Portugal, logo após a legalização do aborto, houve um aumento do número de abortos realizados, porém não ocorreu um aumento equivalente na quantidade de internações devido ao melhor planejamento e programação. Após um aumento inicial, ocorreu uma diminuição no número de abortos e hospitalizações.O mesmo fato aconteceu na Inglaterra e na França (GOLÇALVES-PINHO et al., 2016).

Com base em estudos realizados na Nigéria, verificou-se que as complicações mais frequentes são sangramentos graves, dores e febre logo após o aborto induzido. Além disso, o atraso na busca por atendimento piorou as complicações já existentes. Considerando as informações anteriores, observou-se que o custo para o tratamento de mulheres com tais complicações é, em média, seis vezes maior do que o gasto com os procedimentos para a prática do aborto induzido (PRADA et al., 2015).

Em Kinshasa, a maioria das pacientes tiveram suas complicações tratadas por métodos desatualizados, como dilatação e curetagem. Somente uma pequena parcela das mulheres receberam medicamentos para dor. Diante do exposto, políticas e programas que promovam a legalização do aborto e o uso de anticoncepcionais são necessárias para reduzir as altas taxas de morbimortalidade originadas pelo aborto inseguro (BANKOLE et al., 2018).

A Organização Mundial de Saúde (OMS) estimou que para cada 100.000 abortos inseguros, 30 resultavam em mortesnas regiões de alta renda; já na África Oriental, este número aumentou era de 530. O risco de complicações graves diminuiu ligeiramente com o aumento do nível de escolaridade, pois as mulheres com uma educação melhor possuíam melhores condições financeiras e, consequentemente, eramcapazes de pagar por serviços de aborto induzido mais seguro(KALILANI-PHIRI et al., 2015). 
Mulheres que residem em áreas urbanas podem ter complicações menos graves do que as moram em áreas rurais, pois conseguem atendimento mais rápido e de melhor qualidade. Perante o exposto, foi possível observar que onde o acesso ao aborto seguro é restrito, as mulheres procuram serviços inseguros; daí a necessidade de investimento e garantia de acesso à atenção integral à saúde reprodutiva, incluindo contracepção e aborto seguro (KALILANI-PHIRI et al., 2015).

O presente estudo não é capaz de abordar esse tema de forma integral, pois grande parte das informações necessárias são dependentes da procura de assistência médica que, muitas vezes, é bastante traumática ou até inexistente. Ressalta-se que ainda há muito a se explorar nesse campo.

\section{CONCLUSÃO}

Este estudo possibilitou evidenciar as principais complicações físicas e psicológicas vivenciadas pelas mulheres em consequência da indução do aborto clandestino, levando em consideração a comparação entre as taxas de mortalidade feminina de países que possuem o aborto legalizado e as dos que não possuem. Além da análise do perfil socioeconômico das mulheres que o realizaram e das principais causas que as impulsionaram.

Ao considerar as complicações físicas causadas pela indução do aborto clandestino, destacam-seas hemorragias, infecções, perfuração uterina, sepse e até mesmo o óbito.

Quanto às psicológicas, tem-seconstrangimento, tristeza, sentimento de culpa e estresse emocional.

Contudo, foi visto que tais complicações podem ser diminuídas ou até mesmo evitadasatravés de um acompanhamento hospitalar adequado e assistênciamédica qualificada, tornando-o mais seguro. Desta forma, muitas mulheres teriam uma melhor qualidade de vida pós-aborto. 


\section{REFERÊNCIAS BIBLIOGRÁFICAS}

ADANIKIN, A. I. et al.Maternal near-miss and death associated with abortive pregnancy outcome: a secondary analysis of the Nigeria Near-miss and Maternal Death Survey. BJOG: An International Journal of Obstetrics \& Gynecology, v. 126, ed. S3, p. 33-40, 2019.

AKINLUSI, F. M. et al.Complicated unsafe abortion in a Nigerian teaching hospital: pattern of morbidity and mortality. Journal of Obstetrics and Gynecology, v. 38, n. 7, p. 961-966, 2018.

ARAÚJO, T. V. B. et al. Delays in access to care for abortion-related complications: the experience of women in Northeast Brazil. Cadernos de SaúdePública, v. 34, n. 6, p. 1-40, 2018.

BANKOLE, A. et al.The Severity and Management of Complications Among Postabortion Patients Treated in Kinshasa Health Facilities. International Perspectives on Sexual and Reproductive Health, v. 44, n. 1, p. 1-9, 2018.

DOMINGUES, R. M. S. M. et al. Aborto inseguro no Brasil: revisão sistemática da produção científica, 2008-2018. Cadernos de Saúde Pública, v. 36, supl. 1, p. 1-40, 2020.

GAROFALO, G. et al. Maternal outcomes in first and second trimester termination of pregnancy: which are the risk factors? Journal of Perinatal Medicine, v. 46, n. 4, p. 373-378, 2018.

GOES, E. F. et al.Vulnerabilidade racial e barreiras individuais de mulheres em busca do primeiro atendimento pós-aborto. Cadernos de Saúde Pública, vol.36, n. 1, p. 1-13, 2020.

GONÇALVES-PINHO, M. et al.The impact of a liberalisation law on legally induced abortion hospitalisations. European Journal of Obstetrics e Gynecology and Reproductive Biology, v. 203, p. 142-146, 2016.

HUQ, M. E. et al. Why Abortion is Illegal? Comparison of Legal and llegal Abortion: A Critical Review. Mymensingh Medical Journal, v. 26, n. 4, p. 944-952, 2017.

ISHOSO, D. K. et al. Extent of induced abortions and occurrence of complications in Kinshasa, Democratic Republic of the Congo. Reproductive Health, v. 16, n. 49, p. 1-8,2019.

KALILANI-PHIRI, L. et al. The severity of abortion complications in Malawi. Internacinal Journal of Gynecology e Obstetrics, v. 128, n. 2, p. 160-8, 2015.

KATUASHI, D. I.; TSHEFU, A. K.; COPPIETRS, Y. Analysis of induced abortion-related complications in women admitted to the Kinshasa reference general hospital: a tertiary health facility, Democratic Republic of the Congo. Reproductive Health, v.15, n.123, p. 1-7, 2018.

LABANDERA, A.; GORGOROSO, M.; BRIOZZO, L. Implementations of the risk and harm reduction strategy against unsafe abortion in Uruguay: From a university hospital to the entire country. Internacinal Journal of Gynecology e Obstetrics, v.134, n. S1, p. S7-S11, 2016.

MADEIRO, A. P.; RUFINO, A. C. ADOLEC-Maus-tratos e discriminação na atenção ao aborto induzido: percepção de mulheres em Teresina, Estado do Piauí, Brasil. Ciência e Saúde Coletiva, v. 22, n. 8, p. 2771-2780, 2017.

MADZIYIRE, M. G. et al.Severity and management of postabortion complications among women in Zimbabwe, 2016: a cross-sectional study. British Medical Journal Open, v. 8, n. 2, p. 1-11, 2018. 
MELESE, T. et al. High Levels of Post-Abortion Complication in a Setting Where Abortion Service Is Not Legalizes. Public Libery of Science One, v. 12, n. 1, p. 1-13, 2017.

NAHAR, S. et al. Study on Clinical Presentation and Outcome of Septic Abortion and Its Relationship with Person Inducing Abortion. Mymensingh Medical Journal, v. 26, n. 4, p. 699704, 2017.

PRADA, E. et al. Maternal Near-Miss Due to Unsafe Abortion and Associated Short-Term Health and Socio-Economic Consequences in Nigeria. African Journal of Reproductive Health, v.19, n. 2, p.52-62, 2015.

RATOVOSON, R. et al. Frequency, risk factors, and complications of induced abortion in ten districts of Madagascar: results from a cross-sectional household survey. BMC Womens Health, v. 20 , n. 96 , p. $1-11,2020$.

SELL, S. E. et al. Motivos e significados atribuídos pelas mulheres que vivenciaram o aborto induzido: revisão integrativa. Revista da Escola de Enfermagem da USP vol. 49, n. 3, p. 1-7, 2015.

SOUZA, M. T.; SILVA, M. D.; CARVALHO, R. Integrative review: what is it? How to do it? Einstein (São Paulo), v. 8, n. 1, p.102-106, 2010.

VALADÃO, J. S.; REIS, M. F.; DA ROCHA, F. N. Aborto induzido: o atendimento psicológico em foco. Revista Mosaico, v.10, n. 1, p. 71-77, 2019.

VICENTE, L. F. Aborto por opção da mulher: a experiência portuguesa da implementação da Rede Nacional. Cadernos de Saúde Pública,vol.36, n. 1, p. 1-6, 2020.

VOLKOV, V. G. et al. Abortion in the Structure of Causes of Maternal Mortality. Revista Brasileira de Ginecologia e Obstetrícia, v. 40, n. 6, p. 309-312, 2018.

ZAFAR, H. et al.Low Socioeconomic Status Leading to Unsafe Abortion-related Complications: A Third-world Country Dilemma. The Cureus Journal of Medical Science, v. 10, p. 1-7,2018. 